Laboratorio de Investigación Biomédica Facultad de Medicina, Universidad Católica de Maule, Talca, Chile. 2Departamento de Ciencias Preclínicas, Facultad de Medicina, Universidad

Católica del Maule, Talca Chile.

Unidad de Anatomía Patológica Hospital Regional de Talca, Chile ${ }^{4}$ Centro de Cáncer Gástrico, Hospital Regional de Talca, Chile.

Departamento de Ciencias Clínicas, Facultad de Medicina, Universidad Católica del Maule, Talca, Chile.

Doctor en Ciencias Biológicas.

bMicrobióloga, Máster en Ciencias

Tecnólogo Médico. ¿Bioquímico.

Trabajo financiado por Fondecyt a través del proyecto 1090340

Recibido el 7 de marzo de 2012, aceptado el 12 de agosto de 2013

Correspondencia a: Dr. Erik Morales M

Departamento de Ciencias Preclínicas, Facultad de Medicina, Universidad Católica del Maule, Chile. Teléfono-Fax: 071-2203381. E-mail: emorales@ucm.cl

\section{Lesiones gástricas en pacientes infectados con Helicobacter pylori: expresión de RAGE (receptor de productos de glicosilización avanzada) y otros inmunomarcadores}

\author{
ERIK MORALES M. ${ }^{1,2,3}$, ARMANDO ROJAS R. ${ }^{1, a}$, \\ VALERIA MONASTERIO A., ${ }^{2,3}$, ILEANA GONZÁLEZ B. ${ }^{1,6}$, \\ INGRID FIGUEROA C. ${ }^{3, c}$, BELARMINO MANQUES M. ${ }^{3, c}$, \\ JACQUELINE ROMERO E. ${ }^{1, \mathrm{~d}}$, JORGE LLANOS L., ${ }^{4,5}$, \\ ELIANA VALDÉS M. ${ }^{4,5}$, CECILIA COFRÉ L. ${ }^{4,5}$
}

\section{Expression of RAGE in Helicobacter pylori infested gastric biopsies}

\begin{abstract}
Background: Inflammation is a common phenomenon present in gastric mucosa of patients infected with $H$. pylori. Activation of the RAGE/multiligand axis is thought to be a relevant factor in cancer-mediated inflammation. RAGE is a membrane receptor, belonging to the immunoglobulin family, and the over-expression of RAGE has been associated with increased invasiveness and metastasis generation in different types of cancer, including gastric cancer. Furthermore recent experiences show that the use of its soluble form (sRAGE) or silencing of the gene coding for this receptor could provide therapeutic benefits in cancer. Aim: To evaluate the immunohistochemical expression of RAGE, MUC-1, $\beta$-Catenin free and phosphorylated, Cyclin-D1 and GSK3 in gastric biopsy specimens infected with H. pylori. Material and Methods: Immunohistochemical analysis was carried out in gastric biopsies from 138 patients: 55 with inflammatory injury (no atrophic gastritis), 42 with pre-cancerous conditions (atrophy or intestinal metaplasia) and 41 with dysplastic lesions or in situ adenocarcinoma. Results: There was a high rate of positive RAGE expression in the three groups of biopsies. Biopsies with dysplasia or in situ carcinoma had a significantly higher percentage of RAGE expression than the other groups of biopsies. Conclusions: The increased RAGE expression reported in both dysplasia and incipient cancer support the role of the multiligand/RAGE axis in gastric carcinogenesis.
\end{abstract}

(Rev Med Chile 2013; 141: 1240-1248).

Key words: Carcinogenicity tests; Helicobacter pylori; Stomach neoplasms.
E 1 cáncer gástrico es una de las neoplasias más comunes en el mundo, con más de 988.000 nuevos casos y 736.000 defunciones anualmente ${ }^{1}$. La tasa de mortalidad de esta neoplasia es la segunda más alta a nivel mundial, sólo superada por el cáncer pulmonar. Entre los países afectados, Chile continúa siendo uno de los que presenta mayores tasas de prevalencia ${ }^{1}$. Las mayores tasas de mortalidad por esta neoplasia se presentan en las regiones del Bío Bío, del Maule y de La Araucanía ${ }^{2}$. En la Región del Maule esta tasa es superior a $28 / 100.000$ habitantes $^{3}$.

La infección por Helicobacter pylori es el principal factor de riesgo para el desarrollo de cáncer 
gástrico y lesiones consideradas precursoras de esta neoplasia ${ }^{4}$. A nivel mundial más de $50 \%$ de la población estaría afectada por esta infección ${ }^{5}$ y en Chile $74,5 \%$ de la población entre 20 y 60 años ${ }^{6}$. Esta relación entre infección por $H$. pylori y cáncer gástrico ha sido ampliamente demostrada ${ }^{7}$ y se postula que en más de $80 \%$ de los casos la carcinogenia involucra vías moleculares alteradas por esta bacteria ${ }^{8}$.

Característica fundamental de la infección con H. pylori es la inflamación crónica de la mucosa gástrica, siendo el rol de la inflamación como factor favorecedor del crecimiento tumoral ampliamente reconocido ${ }^{7,9,10}$ y durante los últimos años asociado a la tumorogénesis relacionada al eje multiligando/RAGE ${ }^{11,12}$. RAGE es un receptor de membrana, miembro de la familia de inmunoglobulinas ${ }^{13}$ con homología estructural y funcional con receptores "inmunoglobulina-like"14. A nivel de ligandos, RAGE interactúa principalmente con estructuras tridimensionales, siendo considerado un receptor de patrones moleculares (similar receptores tipo Tolls) lo que explicaría su unión a varias moléculas, endógenas o exógenas ${ }^{15}$.

RAGE se vincula a una serie de procesos patológicos asociados a inflamación, como diabetes, cáncer, enfermedades neurodegenerativas e insuficiencia renal y cardiaca ${ }^{16,17}$. En neoplasias, la expresión de RAGE se ha descrito en cerebro, mama, colorrecto, pulmón, próstata, oral y ovario, también en linfomas y melanomas ${ }^{18}$. En cáncer gástrico, la sobreexpresión de RAGE ha sido asociada a mayor invasión y metástasis ${ }^{19}$. Además algunos polimorfismos del receptor otorgarían mayor susceptibilidad al desarrollo y mayor agresividad de dicha neoplasia ${ }^{20}$. A la fecha existe consenso en que RAGE mantendría un estado inflamatorio que estimula la fase de promoción de la carcinogenia y el desarrollo tumoral ${ }^{21}$. Se ha propuesto también que esta molécula mediaría la activación de procesos claves que promoverían resistencia a los estímulos apoptóticos e hipoxia, interfiriendo con la inmunidad antitumoral y estimulando la angiogénesis e invasión ${ }^{11}$.

A nivel de señalización intracelular, numerosos mecanismos han sido involucrados en la carcinogenia gástrica y, entre estos, la expresión de genes que controlan la proliferación celular vinculados a las vías Wnt, NF-kB y Stat3, presumiblemente con transcripción de genes diana comunes ${ }^{22}$. Stat 3 y NF-kB pertenecen a vías moleculares que pueden ser activadas por la cascada inflamatoria asociada a la estimulación de $\mathrm{RAGE}^{11}$ y en carcinomas dicha activación también ocurriría por la sobreexpresión de la mucina de membrana MUC- $1^{23}$. $\mathrm{Si}$ bien la función canónica de estas mucinas es proteger epitelios vulnerables ${ }^{40}$, en la actualidad autores sugieren que la interacción de MUC-1 con H. pylori podría iniciar una serie de cascadas de transducción en el epitelio glandular ${ }^{23}$ y estudios en cáncer gástrico relacionan su sobreexpresión con un peor pronóstico ${ }^{24-26}$. Componentes de la vía Wnt como $\beta$-catenina y GSK3 $\beta$ también han sido reconocidos como factores vinculantes entre cáncer e inflamación ${ }^{27,28}$ y se ha observado que entre los resultados de su activación existe transcripción de ciclina-D1 ${ }^{29,30}$ un importante regulador del ciclo celular frecuentemente sobreexpresado en los cánceres ${ }^{31}$.

Según lo expuesto; alta prevalencia local del cáncer gástrico, altas tasas de infección por $H$. pylori y el reciente rol descrito para RAGE en la carcinogenia vinculada a inflamación, resulta muy necesario desarrollar un estudio local que aborde la expresión en mucosa gástrica de RAGE, MUC-1 y algunas moléculas de conocida participación en la carcinogenia gástrica ( $\beta$-catenina, ciclina-D1 y GSK3 $\beta$ ).

\section{Material y Método}

\section{Material evaluado y criterios de selección}

Como parte del estudio del eje AGE/RAGE en la patogénesis de la infección por $H$. pylori, el presente trabajo evaluó especímenes de biopsias gástricas positivas para esta infección correspondientes a 138 pacientes sintomáticos con indicación clínica de endoscopia digestiva alta (Unidad de Cáncer Gástrico del Hospital Regional de Talca, noviembre de 2010 a marzo de 2011) y sin tratamiento (inhibidores de la bomba de protones) o antiinflamatorios no esteroidales en las cuatro semanas previas al examen. En 97 de casos la presencia de $H$. pylori fue pesquisada al momento de la endoscopia mediante test rápido de ureasa y confirmada mediante técnica de reacción de polimerasa en cadena. En los casos en que no fue posible realizar técnicas moleculares, la presencia del bacilo fue demostrada mediante tinción histoquímica (Diff). Confirmada la presencia bacteriana, los casos fueron clasificados 
según criterios histopatológicos: clasificaciones de Sydney para gastritis ${ }^{32}$ y de Viena para lesiones neoplásicas gastrointestinales ${ }^{33}$. A partir de esta evaluación se estructuraron tres grupos comparables representativos de la secuencia descrita para carcinogenia gástrica: i) "Gastritis crónica" (no atrófica), ii) "Condiciones preneoplásicas" (atrofia y/o metaplasia intestinal) y iii) "Displasia o neoplasia in situ" (displasia epitelial glandular o adenocarcinoma in situ $)^{34}$.

\section{Protocolo de procesamiento de muestras}

Las muestras fueron fijadas en formalina neutra $10 \%$ (durante $24 \mathrm{~h}$ ), deshidratadas, aclaradas e impregnadas. Inclusión en parafina (Merck, fusión $56^{\circ} \mathrm{C}$ ). Se obtuvo cortes de $3 \mu \mathrm{m}$, secados $\left(65^{\circ} \mathrm{C}\right.$ durante $\left.45 \mathrm{~min}\right)$ y desparafinados (xilol, alcohol $100^{\circ}$ y alcohol $95^{\circ}$ ). Luego hidratación y recuperación antigénica (Antigen Unmasking Solution VECTOR 1M) con microondas (20 min). Se ocupó Peroxidase Detection System de Novocastra, incubando con anticuerpo primario (estandarización y especificaciones técnicas del proveedor, $\beta$-catenina 12F7, 1:500; Mucina-1E29, 1:500; RAGE A11, 1:200; GSK-3ß H-76, 1:200; p- $\beta$-catenina Ser37, 1:100; Ciclina-D1 A-12, 1:100, anticuerpos Santa Cruz Biotechnology). Lavados con buffer PBS (Winkler) y cortes incubados $3 \mathrm{~min}$ con DAB (Novocastra) para revelar la expresión. Medio de montaje Shandon.

\section{Evaluación de la expresión inmunohistoquímica}

Según protocolo endoscópico, cada caso incluyó muestras de mucosa antral, especializada y de cualquier otra zona en que se identificó alguna alteración. Sin perjuicio de lo anterior, en función del objetivo del estudio el análisis de inmunoexpresión privilegió la mucosa con alteraciones histopatológicas o con presencia morfológica de Helicobacter pylori. Para evaluar la inmunotinción se consideró tanto la presencia de expresión (positiva o negativa) como el patrón de inmunomarcación (citoplasmático, membrana plasmática o nuclear) del epitelio glandular. Para RAGE además se evaluó niveles de expresión (bajo o G1 = marcación débil $<75 \%$ o intensa en $<25 \%$ de las células, y alto o $\mathrm{G} 2$ = marcación débil en más de $75 \%$ o intensa en más de $25 \%$ de las células) ${ }^{35}$. Todos los casos fueron evaluados por dos observadores de manera independiente, las diferencias de estimación $(<10 \%)$ fueron resueltas mediante consenso. Para clasificar un caso como negativo, se exigió positividad en mucosa vecina a la evaluada (control interno) o en el control positivo externo. Casos no concluyentes fueron reprocesados para segunda evaluación.

\section{Análisis estadístico}

El análisis estadístico se realizó con programa informático SPSS (PASW Statistics 18). La positividad para la inmunomarcación en los tres grupos estudiados fue analizada utilizando la herramienta de prueba estadística ANOVA que permite comparar simultáneamente medias de más de dos grupos de estudio.

\section{Resultados}

Características clínico-patológicas de los casos estudiados

Las principales características clínico-patológicas se resumen en la Tabla 1.

Tabla 1. Características clínico-patológicas

\begin{tabular}{|c|c|c|c|}
\hline Características & $\begin{array}{l}\text { Gastritis crónica } \\
(\mathrm{GC}, \mathrm{n}=\mathbf{5 5})\end{array}$ & $\begin{array}{l}\text { Condiciones preneoplásicas } \\
(\mathrm{CP}, \mathrm{n}=\mathbf{4 2})\end{array}$ & $\begin{array}{l}\text { Displasia/CIS } \\
(\mathrm{D}-\mathrm{CIS}, \mathrm{n}=41)\end{array}$ \\
\hline Sexo (H: M) & 19: 36 & $16: 26$ & $24-17$ \\
\hline $\begin{array}{l}\text { Edad (años) } \\
\text { Rango } \\
\text { Promedio }\end{array}$ & $\begin{array}{l}22-78 \\
48\end{array}$ & $\begin{array}{l}20-75 \\
52\end{array}$ & $\begin{array}{l}20-77 \\
61\end{array}$ \\
\hline $\begin{array}{l}\text { Endoscopia } \\
\text { Sin lesión } \\
\text { Con lesión }\end{array}$ & $\begin{array}{l}30(54 \%) \\
25(46 \%)\end{array}$ & $\begin{array}{l}16(38 \%) \\
26(62 \%)\end{array}$ & $\begin{array}{l}3(5 \%) \\
39(95 \%)\end{array}$ \\
\hline
\end{tabular}


Lesiones gástricas asociadas a H. Pylori: RAGE y otros inmunomarcadores - E. Morales et al

\section{Resultados de la inmunomarcación}

Las Figuras la y $1 \mathrm{~b}$ muestran focos de expresión de RAGE considerados positivos de acuerdo a los criterios antes citados. Para este marcador se observó tanto positividad a nivel de membrana plasmática/citoplasma (MP/C) como nuclear $(\mathrm{N})$.

La Tabla 2 y la Figura 2 muestran los porcentajes de casos positivos a la inmunomarcación de RAGE. Al comparar estos porcentajes de casos positivos en los tres grupos estudiados ("Gastritis", "Condiciones preneoplásicas" y "Displasia/CIS") se observó diferencia estadísticamente significativa (nivel $\mathrm{p}<0,05$ ) para la inmunomarcación de alto grado (G2) tanto a nivel de membrana plasmática/ citoplasma $(23,6 \%, 40,5 \%$ y $63,4 \%$, respectivamente) como a nivel nuclear $(23,6 \%, 38,1 \%$ y $58,5 \%$, respectivamente) (Figura 2).

La Tabla 3 muestra los porcentajes de casos positivos para inmunoexpresión a MUC-1, $\beta$-catenina, $\beta$-catenina fosforilada, ciclina-D1 y GSK3 $\beta$. Para MUC-1 se observó positividad a nivel de membrana plasmática (MP)/citoplasma (C) y en membrana plasmática apical (MA). Para $\beta$-catenina se observó positividad de membrana plasmática (MP)/citoplasma (C) y nuclear $(\mathrm{N})$. Para $\beta$-catenina fosforilada, ciclina-D1 y GSK3 $\beta$ se observó positividad de citoplasma (C) y nuclear $(\mathrm{N})$. Al comparar los porcentajes de casos posi-
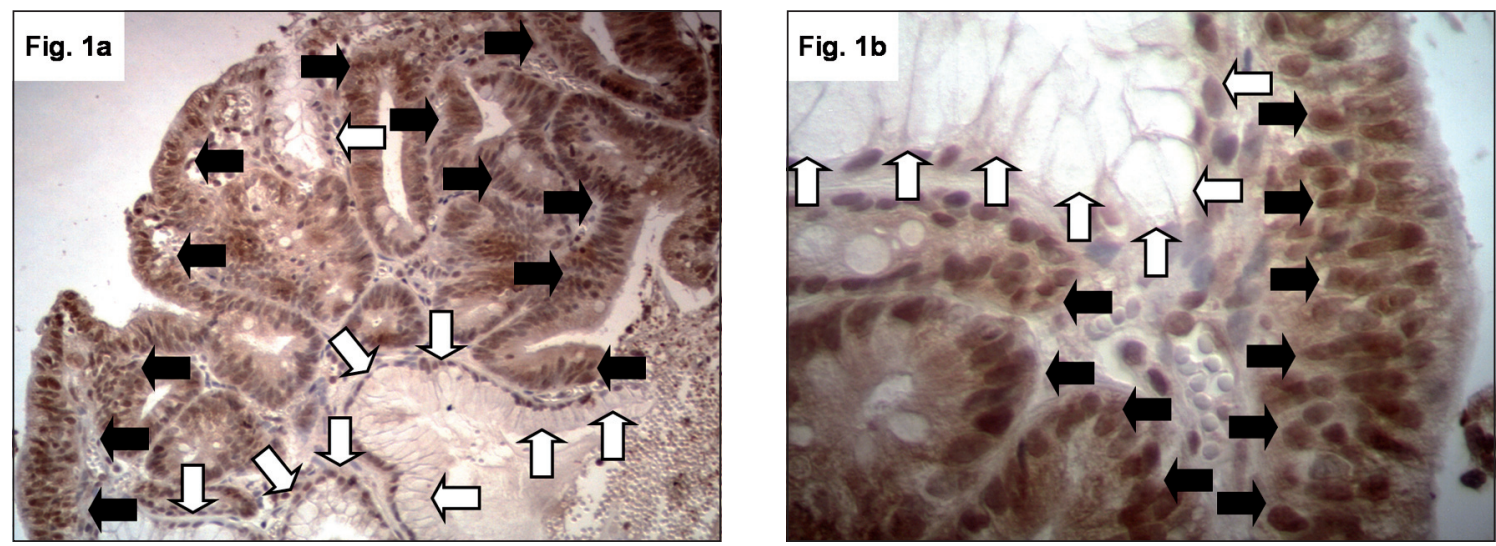

Figura 1. Positividad a RAGE. Mucosa gástrica con inmunomarcación para RAGE. Tanto en microfotografía con aumento 4x (Figura 1a) como en microfotografía con aumento 10x (Figura 1b) se observan zonas de epitelio glandular con caracteres displásicos (desorden arquitectural, amontonamiento nuclear, pérdida de polaridad e hipercromasia nuclear) los que además muestran positividad a la inmunoexpresión citoplasmática y nuclear para RAGE (flechas negras). En ambas microfotografías se observan también zonas de epitelio glandular normotípico o con leves cambios reactivos, negativas o de escasa positivad para el receptor (flechas blancas).

Tabla 2. Positividad para RAGE

\begin{tabular}{|c|c|c|c|c|c|c|c|c|}
\hline \multirow{3}{*}{$\begin{array}{l}\text { Marcación } \\
\text { Localización } \\
\text { MP/C }\end{array}$} & \multirow{3}{*}{$\begin{array}{l}\text { Nivel de } \\
\text { positividad } \\
\text { Negativos }\end{array}$} & \multicolumn{6}{|c|}{ Grupo de estudio } & \multirow[t]{2}{*}{$p<0,05$} \\
\hline & & \multicolumn{2}{|c|}{$\begin{array}{l}\text { Gastritis crónica } \\
\qquad(\mathrm{GC}, \mathrm{n}=55)\end{array}$} & \multicolumn{2}{|c|}{$\begin{array}{l}\text { Condiciones preneoplásicas } \\
\qquad(\mathrm{CP}, \mathrm{n}=\mathbf{4 2})\end{array}$} & \multicolumn{2}{|c|}{$\begin{array}{l}\text { Displasia - CIS } \\
(D-C I S, n=41)\end{array}$} & \\
\hline & & 22 & $(40,0 \%)$ & 11 & $(26,2 \%)$ & 10 & $(24,4 \%)$ & \\
\hline & Bajo (G1) & 20 & $(36,4 \%)$ & 14 & $(33,3 \%)$ & 5 & $(12,2 \%)$ & \\
\hline & Alto (G2) & 13 & $(23,6 \%)$ & 17 & $(40,5 \%)$ & 26 & $(63,4 \%)$ & $(*)$ \\
\hline \multirow[t]{4}{*}{ N } & Negativos & 21 & $(38,2 \%)$ & 10 & $(23,8 \%)$ & 9 & $(22,0 \%)$ & \\
\hline & Bajo (G1) & 21 & $(38,2 \%)$ & 16 & $(38,1 \%)$ & 8 & $(19,5 \%)$ & \\
\hline & Alto (G2) & 13 & $(23,6 \%)$ & 16 & $(38,1 \%)$ & 24 & $(58,5 \%)$ & $(*)$ \\
\hline & & 55 & $(100 \%)$ & 42 & $(100 \%)$ & 41 & $(100 \%)$ & \\
\hline
\end{tabular}

$\mathrm{C}=$ Citoplasma; $\mathrm{MA}=$ Membrana apical; $\mathrm{MP}=$ Membrana plasmática; $\mathrm{N}=$ Núcleo. 


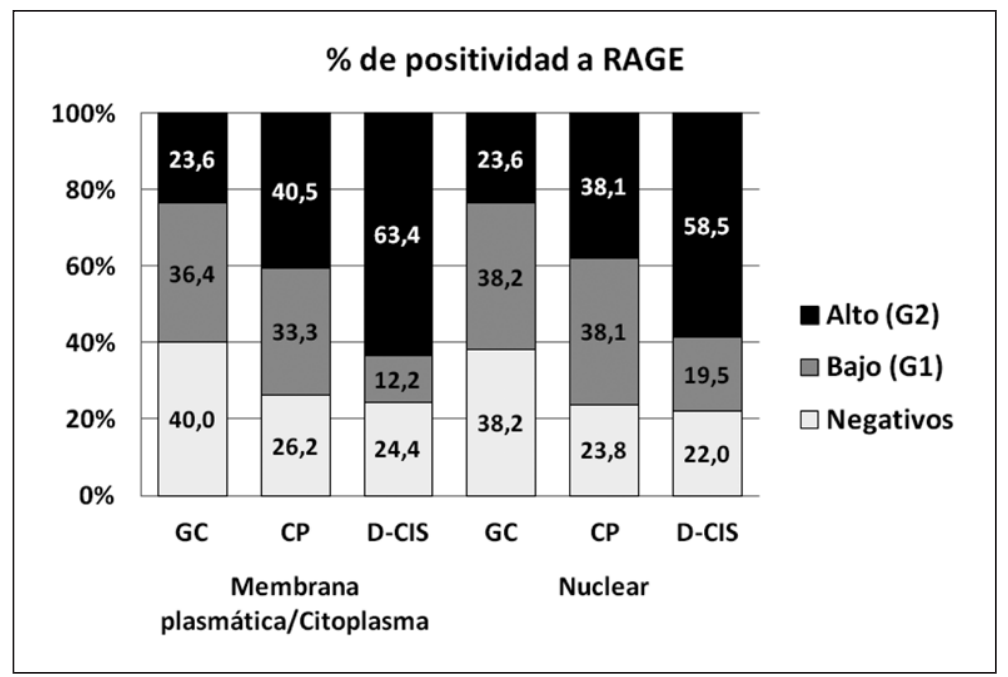

Figura 2. Porcentajes de positividad para RAGE. Se muestra gráficos con porcentajes de casos positivos a la inmunomarcación de RAGE. Se observa un aumento de la expresión en el grupo Displasia-CIS, tanto a nivel de membrana como nuclear. Al analizar con mayor detalle la expresión de la inmunomarcación también se observa que en el grupo de Displasia-CIS hay un importante aumento del porcentaje de casos que tienen altos niveles de positividad (G2). C = Citoplasma; MA = Membrana Apical; MP = Membrana Plasmática; N = Núcleo; GC = Gastritis crónica, $C P=$ Condiciones Preneoplásicas; $\mathrm{D}-\mathrm{ClS}=$ Displasia/Adenocarcinoma in situ.

Tabla 3. Positividad a otros marcadores

\begin{tabular}{|c|c|c|c|c|c|c|c|c|}
\hline \multirow{3}{*}{$\begin{array}{l}\text { Marcación } \\
\text { Marcador } \\
\text { MUC-1 }\end{array}$} & \multirow{3}{*}{$\begin{array}{l}\text { Localización } \\
\text { MA } \\
\text { MP/C }\end{array}$} & \multirow{2}{*}{\multicolumn{4}{|c|}{$\begin{array}{l}\text { Grupo de estudio } \\
\text { Condiciones } \\
\text { preneoplásicas } \\
(\mathrm{CP}, \mathrm{n}=\mathbf{4 2})\end{array}$}} & \multirow{2}{*}{\multicolumn{2}{|c|}{$\begin{array}{c}\text { Displasia/ CIS } \\
\text { (D-CIS, } n=41)\end{array}$}} & \multirow{3}{*}{$\begin{array}{c}\text { p }<0,05 \\
\left(^{*}\right)\end{array}$} \\
\hline & & & & & & & & \\
\hline & & $\begin{array}{l}40 \\
49\end{array}$ & $\begin{array}{l}(72,7 \%) \\
(89,1 \%)\end{array}$ & $\begin{array}{l}25 \\
39\end{array}$ & $\begin{array}{l}(59,5 \%) \\
(92,9 \%)\end{array}$ & $\begin{array}{r}9 \\
34\end{array}$ & $\begin{array}{l}(22 \%) \\
(82,9 \%)\end{array}$ & \\
\hline$\beta$ Catenina & $\begin{array}{l}\mathrm{N} \\
\mathrm{MP} / \mathrm{C}\end{array}$ & $\begin{array}{c}9 \\
55\end{array}$ & $\begin{array}{l}(16,4 \%) \\
(100 \%)\end{array}$ & $\begin{array}{l}12 \\
42\end{array}$ & $\begin{array}{l}(28,6 \%) \\
(100 \%)\end{array}$ & $\begin{array}{l}26 \\
28\end{array}$ & $\begin{array}{l}(63,4 \%) \\
(68,3 \%)\end{array}$ & $\begin{array}{l}(*) \\
\left(^{*}\right)\end{array}$ \\
\hline p $\beta$ Catenina & $\begin{array}{l}\mathrm{N} \\
\mathrm{C}\end{array}$ & $\begin{array}{r}33 \\
7\end{array}$ & $\begin{array}{l}(60 \%) \\
(12,7 \%)\end{array}$ & $\begin{array}{r}25 \\
8\end{array}$ & $\begin{array}{l}(59,5 \%) \\
(19,0 \%)\end{array}$ & $\begin{array}{r}34 \\
0\end{array}$ & $\begin{array}{c}(82,9 \%) \\
(0 \%)\end{array}$ & $\left({ }^{\star}\right)$ \\
\hline GSK3 $\beta$ & $\begin{array}{l}\mathrm{N} \\
\mathrm{C}\end{array}$ & $\begin{array}{l}35 \\
37\end{array}$ & $\begin{array}{l}(63,6 \%) \\
(67,3 \%)\end{array}$ & $\begin{array}{l}33 \\
34\end{array}$ & $\begin{array}{l}(78,6 \%) \\
(81 \%)\end{array}$ & $\begin{array}{l}23 \\
38\end{array}$ & $\begin{array}{l}(56,1 \%) \\
(92,7 \%)\end{array}$ & $\left(^{*}\right)$ \\
\hline Ciclina D1 & $\begin{array}{l}N \\
C\end{array}$ & $\begin{array}{l}38 \\
37\end{array}$ & $\begin{array}{l}(69,1 \%) \\
(67,3 \%)\end{array}$ & $\begin{array}{l}29 \\
33\end{array}$ & $\begin{array}{l}(69 \%) \\
(78,6 \%)\end{array}$ & $\begin{array}{l}36 \\
26\end{array}$ & $\begin{array}{l}(87,8 \%) \\
(63,4 \%)\end{array}$ & $\left({ }^{\star}\right)$ \\
\hline
\end{tabular}

$\mathrm{C}=$ Citoplasma; $\mathrm{MA}=$ Membrana apical; $\mathrm{MP}=$ Membrana plasmática; $\mathrm{N}=$ Núcleo.

tivos para inmunomarcación en los tres grupos estudiados ("Gastritis", "Condiciones preneoplásicas" y "Displasia/CIS") se observó diferencia estadísticamente significativa (nivel p $<0,05$ ) en la inmunomarcación apical de MUC-1, nuclear y de membrana plasmática/citoplasma de $\beta$-catenina, nuclear de $\beta$-catenina fosforilada, citoplasmática de GSK3 $\beta$ y nuclear de ciclina-D1.

\section{Discusión}

El cáncer gástrico es una de las neoplasias más comunes en el mundo ${ }^{1}$ y la infección por Helico- bacter pylori es el principal factor de riesgo para su desarrollo ${ }^{4}$. Una característica fundamental de la infección gástrica con $H$. pylori es el desarrollo de un proceso inflamatorio crónico mucoso y el rol de la inflamación como factor favorecedor del crecimiento neoplásico ha sido ampliamente reconocido ${ }^{7,9,10}$. En relación con este vínculo entre inflamación y proliferación neoplásica se postula que la activación de RAGE contribuiría de forma importante al proceso carcinogénico ${ }^{11,12}$, lo que estaría respaldado por el hallazgo de sobreexpresión del receptor en numerosas neoplasias (cerebro, mama, colorrecto, pulmón, próstata, oral, ovario, 
etc. $)^{18}$. A nivel gástrico, la sobreexpresión de RAGE no sólo ha sido observada en cáncer (asociada a un mayor nivel de invasión y generación de metástasis ${ }^{19}$, también ha sido observada en procesos inflamatorios y particularmente participando de la adherencia de $H$. pylori al epitelio glandular de la mucosa gástrica ${ }^{36}$.

Los resultados observados en el presente estudio, caracterizados por un alto porcentaje de positividad de RAGE en los tres grupos de estudio (gastritis crónica, condiciones preneoplásicas y displasia/CIS) parecen ratificar desde el reconocimiento morfológico mediante técnica inmunohistoquímica, la sospecha de que este receptor desarrollaría un importante papel en la patogenia de la injuria gástrica en ambiente inflamatorio secundario a infección por $H$. pylori. Además, el hallazgo de un mayor porcentaje de casos positivos en el grupo "Displasia/CIS" y particularmente el de una mayor cantidad de casos con altos niveles de expresión del receptor en este grupo (con diferencia estadísticamente significativa a nivel $\mathrm{p}<0,005)$ sugieren que la sobreexpresión de este receptor jugaría un particular rol en la transformación neoplásica del epitelio glandular gástrico.

La expresión de RAGE fue observada a nivel de membrana plasmática/citoplasma como lo referencia la mayor parte de la literatura ${ }^{37,38}$, y también a nivel nuclear. La expresión nuclear e RAGE es un hallazgo pocas veces descrito ${ }^{39}$ y un desafío en su interpretación. $\mathrm{Al}$ respecto, autores sugieren que como resultado del clivaje durante la proteólisis intramembrana del receptor (mediado por calcio y enzimas metaloproteinasas y secretasa) existiría liberación de su dominio intracelular al espacio citosólico/nuclear ${ }^{40,41}$. Otros sugieren que el ligando S100 podría ser responsable de retener a RAGE en estas localizaciones ${ }^{40}$.

A nivel de señalización intracelular, tanto RAGE $^{11}$ como MUC- ${ }^{23}$ son reconocidos como activadores de vías que controlan proliferación celular e importantes en carcinogenia gástrica (como Wnt, NF-kB y Stat3) ${ }^{22}$. Componentes de la vía Wnt como $\beta$-catenina y GSK3 $\beta$ serían factores vinculantes entre cáncer e inflamación ${ }^{27,28}$ y activadores de la transcripción de ciclina-D129,30 un importante regulador del ciclo celular sobreexpresado en cáncer ${ }^{31}$. Los resultados observados en el presente estudio: alto porcentaje de inmunomarcación a nivel de membrana plasmática/ citoplasma para MUC-1 como el mayor porcentaje de casos positivos en el grupo displasia/CIS a nivel nuclear para $\beta$-catenina y $\beta$-catenina fosforilada, citoplasmático y nuclear para ciclina-D1 y citoplasmático para GSK3 $\beta$ son coherentes con lo expuesto y concordantes con la literatura ${ }^{42-48}$. El caso de MUC-1 es particularmente interesante ya que su unión con $H$. pylori potenciaría la activación de las señales de transducción de vías que controlan el crecimiento celular ${ }^{23}$. La marcada disminución de la inmunomarcación apical para MUC-1 en el grupo de lesiones displásicas/CIS también concuerda con lo descrito por la literatura ${ }^{42,43}$ y podría deberse a pérdida de caracteres de especialización asociada al fenotipo displásico.

Junto con la comprensión de la participación de RAGE en la patogenia de la injuria celular en ambientes inflamatorios y su relación con la carcinogenia, en la actualidad las investigaciones también están enfocadas en los alcances diagnósticos y terapéuticos de esta molécula ${ }^{49-51}$. En este sentido, se han desarrollado propuestas en relación a la utilidad de la forma soluble de RAGE (sRAGE) ya que inhibiría los efectos patológicos mediados por la sobreexpresión del receptor ${ }^{49}$. Además, resultados sugieren que los niveles de sRAGE son un buen biomarcador de progresión de enfermedad en algunos tipos de cáncer ${ }^{49,50}$. Otros estudios han logrado silenciamiento de la expresión de RAGE (mediante RNAi) en neoplasias prostáticas y sugieren que podría transformarse en una estrategia alternativa para tratar el cáncer de próstata ${ }^{49}$. Experiencias similares en cáncer de colorectal sugieren que el beneficio del silenciamiento de RAGE estaría mediado de forma importante por una reducción de la angiogenia tumoral ${ }^{52}$.

En conclusión, el estudio desarrollado permitió evaluar la inmunoexpresión de RAGE y moléculas relevantes en carcinogenia gástrica en mucosa con inflamación crónica, condiciones preneoplásicas (atrofia/metaplasia intestinal) y con lesiones preneoplásicas/neoplasia in situ, desarrolladas en un ambiente inflamatorio secundario a infección por $H$. pylori. Los resultados obtenidos son concordantes con lo descrito por la literatura respecto de las variaciones en la expresión de moléculas involucradas en la transformación maligna de la mucosa gástrica (MUC-1, $\beta$-catenina, $\beta$-catenina fosforilada, GSK-3 $\beta$ y ciclina-D1). El alto porcentaje de casos positivos para la inmunoexpresión de RAGE observada en los tres grupos de estudio sugiere que el eje multiligando/RAGE sería 
relevante en la patogenia de lesiones de mucosa gástrica en contextos inflamatorios por infección con $H$. pylori. La mayor expresión observada en lesiones displásicas/CIS avala las propuestas de que este eje también jugaría un particular rol en la transformación maligna del epitelio glandular gástrico. La observación de inmunoexpresión de RAGE a nivel nuclear es un hecho infrecuente que requiere ser corroborado con otros estudios que permitan confirmar su presencia a este nivel y evaluar el significado funcional de dicho hallazgo. Experiencias muestran que la utilización de RAGE o su forma soluble (sRAGE) tendrían utilidad en el seguimiento de neoplasias y que el silenciamiento del gen que codifica para este receptor podría aportar beneficios terapéuticos. Todo lo anterior sugiere la necesidad de profundizar en el conocimiento de la relación entre RAGE, inflamación y cáncer gástrico.

Agradecimientos: Este trabajo fue financiado por Fondecyt a través del proyecto 1090340.

\section{Referencias}

1. GLOBOCAN 2008, IARC, http://globocan.iarc.fr/. [Consultado el 20 de noviembre de 2011].

2. Icaza NMG, Núñez FML, Torres AFJ, Díaz SL, Varela G, David E. Distribución geográfica de mortalidad por tumores malignos de estómago, tráquea, bronquios y pulmón, Chile 1997-2000. Rev Med Chile 2007; 135: 1397-140. Disponible en: www.scielo.cl [Consultado el 05 de enero de 2012].

3. MINSAL, http://deis.minsal.cl/vitales/Mortalidad_causa/ Chile.htm. [Consultado el 20 de diciembre de 2011].

4. Hamilton S. Pathology and Genetics. Tumours of the Digestive System. WHO Classification of Tumours. Hamilton S, Aaltonen L, editors. Lyon, France: IARC Press; 2000. p. 40.

5. Danesh J. Helicobacter pylori infection and gastric cancer: systematic review of the epidemiological studies. Alimentary Pharmacology and Therapeutics 1999; 13: 851-6.

6. Ferreccio C, Rollán A, Harris PR, Serrano C, Gederlini A, Margozzini P, et al. Gastric cancer is related to early Helicobacter pylori infection in a high-prevalence country. Cancer Epidemiology Biomarkers and Prevention 2007; 16: 662-7.

7. Bornschein J, Kandulski A, Selgrad M, Malfertheiner P. From gastric inflammation to gastric cancer. Dig Dis 2010; 28 (4-5): 609-14.
8. Houghton J, Wang TC. Helicobacter pylori and gastric cancer: a new paradigm for inflammation-associated epithelial cancers. Gastroenterology 2005; 128: 1567-78.

9. Blakwill F, Montovani A. Inflammation and cancer: back to Virchow? Lancet 2001; 237: 539-45.

10. Mantovani A, Allavena P, Sica A, Balkwill F. Cancerrelated inflammation. Nature 2008, 454, 436-44.

11. Rojas A, Figueroa H, Morales E. Fueling inflammation at tumor microenvironment: the role of multiligand/rage axis. Carcinogenesis 2010; 31: 334-41.

12. Riehl A, Nemeth J, Angel P, Hess J. The receptor RAGE: Bridging inflammation and cancer. Cell Communication and Signaling 2009, 7: 12. Disponible en: www. biosignaling.com [Consultado el 20 de diciembre de 2011].

13. Basta G. Receptor for advanced glycation endproducts and atherosclerosis: from basic mechanisms to clinical implications. Atherosclerosis 2008; 19: 9-21.

14. Schmidt AM, Vianna M, Gerlach M, Brett J, Ryan J, Kao $\mathrm{J}$, et al. Isolation and characterization of two binding proteins for advanced glycosylation end products from bovine lung which are present on the endothelial cell surface. J Biol Chem 1992; 267: 14987-97.

15. Bierhaus A, Humpert PM, Morcos M, Wendt T, Chavakis T, Arnold B, et al. Understanding RAGE, the receptor for advanced glycation end products. J Mol Med 2005; 83: 876-86.

16. Yan SF, Ramasamy R, Schmidt AM. Mechanisms of disease: advanced glycation endproducts and their receptor in inflammation and diabetes complications. Nat Clin Pract Endocrinol Metab 2008; 4: 285-93.

17. Rojas A, Mercadal E, Figueroa H, Morales MA. Advanced Glycation and ROS: a link between diabetes and heart failure. Curr Vasc Pharmacol 2008; 6: 44-51.

18. Logsdon CD, Fuentes MK, Huang EH, Arumugam T, RAGE and RAGE ligands in cancer. Curr Mol Med 2007; 7: 777-89.

19. Kuniyasu H, Oue N, Wakikawa A, Shigeishi H, Matsutani N, Kuraoka K, et al. Expression of receptors for advanced glycation end-products (RAGE) is closely associated with the invasive and metastatic activity of gastric cancer. Journal of Pathology 2002; 196: 163-70.

20. Haijuan G, Li Y, Qingmin S, Bo Z, Naping T, Rihong C, et al. Gly82 Ser Polymorphism of the Receptor for Advanced Glycation End Products Is Associated with an Increased Risk of Gastric Cancer in a Chinese Population, Clin Cancer Res 2008; 14: 3627-32.

21. Gebhardt C, Riehl A, Durchdewald M, Németh J, Fürstenberger G, Müller-Decker K, et al. RAGE signaling sustains inflammation and promotes tumor development. The Journal of Experimental Medicine 2008; 205: 
275-85.

22. Jing-Chun H, Kai-Li Z, Xiao-Yan C, Hai-Feng J, QingYou K, Yuan S, et al. Expression of seven gastric cancerassociated genes and its relevance for Wnt, NF- $\kappa \mathrm{B}$ and Stat3 signaling. Acta Pathologia, Microbiologica et Immunologica Scandinavica 2007; 115 (12): 1331-43.

23. Lindén S, Mahdavi J, Hedenbro J, Borén T, Carlstedt I. Effects of $\mathrm{pH}$ on Helicobacter pylori binding to human gastric mucins: identification of binding to non-MUC5AC mucins. Biochemical Journal 2005; 384: 263-70.

24. Ahmad R, Raina D, Trivedi V, Ren J, Rajabi H, Kharbanda S, et al. MUC1 oncoprotein activates the IkappaB kinase beta complex and constitutive NF-kappaB signalling. Nature Cell Biology 2007; 9: 1419-27.

25. Utsunomiya T, Yonezawa S, Sakamoto H, Kitamura H, Hokita S, Aiko T, et al. Expression of MUC1 and MUC2 mucins in gastric carcinomas: its relationship with the prognosis of the patients. Clinical Cancer Research 1998; 4: 2605-14.

26. Wei X, Xu H, Kufe D. Human Mucin 1 oncoprotein represses transcription of the p53 tumor suppressor gene. Cancer Research 2007; 67: 1853-8.

27. George SJ. Wnt pathway: a new role in regulation of inflammation. Arteriosclerosis Thrombosis and Vascular Biology 2008; 28: 400-2.

28. Kikuchi A, Yamamoto H. Tumor formation due to abnormalities in the beta-catenin-independent pathway of Wnt signaling. Cancer Science 2008; 99: 202-8.

29. Cadigan KM, Nusse R. Wnt signaling: a common theme in animal development. Genes and Development 1997; 11: 3286-305.

30. Yong HY, Moon A. Roles of calcium-binding proteins, S100A8 and S100A9, in invasive phenotype of human gastric cancer cells. Archives of Pharmacal Research 2007; 30: 75-81.

31. Kyong Kim J, Diehl JA. Nuclear cyclin D1: An oncogenic driver in human cancer. J Cell Physiol 2009; 220 (2): 292-6.

32. Dixon M, Genta R, Yardley J, Correa P, et al. Classification and Grading of Gastritis. The Updated Sydney System. The American Journal of Surgical Pathology 1996; 20 (10): 1161-81.

33. Schlemper R, Riddell R, Kato Y, Borchard F, Cooper H, Dawsey S, et al. The Vienna Classification of gastrointestinal epithelial neoplasia. Gut 2000; 47: 251-5.

34. Correa P and Chen VW. Gastric Cancer. Cancer Surv 1994; 19-20: 55-76.

35. Guzmán P, Araya J, Villaseca M, Roa I, Melo A, Muñoz $\mathrm{S}$, et al. Expresión inmunohistoquímica del complejo E-caderina-catenina en cáncer gástrico. Relación con variables clínico-morfológicas y sobrevida de pacientes.
Rev Med Chile 2006; 134: 1002-9.

36. Rojas A, González I, Rodríguez B, Romero J, Figueroa H, Llanos J, et al. Evidence of involvement of the receptor for advanced glycation end-products (RAGE) in the adhesion of Helicobacter pylori to gastric epithelial cells. Microbes Infect 2011; 13 (10): 818-23. Epub 2011 May 12.

37. Sasahira T, Kirita T, Bhawal UK, Yamamoto K, Ohmori $\mathrm{H}$, Fujii K, et al. Receptor for advanced glycation end products (RAGE) is important in the prediction of recurrence in human oral squamous cell carcinoma. Histopathology 2007; 51: 166-72.

38. Tateno T, Ueno S, Hiwatashi K, Matsumoto M, Okumura H, Setoyama T, et al. Expression of Receptor for Advanced Glycation End Products (RAGE) is Related to Prognosis in Patients with Esophageal Squamous Cell Carcinoma. Ann Surg Oncol 2009; 16: 440-6.

39. Diamanti-Kandarakis E, Piperi C, Papavassiliou AG, Patsouris E, Korkolopoulou P, Panidis D, et al. Immunohistochemical localization of advanced glycation end-products (AGEs) and their receptor (RAGE) in polycystic and normal ovaries, Histochem Cell Biol 2007; 127: 581-9.

40. Sparvero LJ, Asafu-Adjei D, Kang R, Tang D, Amin N, Im J, et al. RAGE (Receptor for Advanced Glycation Endproducts), RAGE Ligands, and their role in Cancer and Inflammation. Journal of Translational Medicine 2009; 7: 17.

41. Galichet A, Weibel M, Heizmann CW. Calcium-regulated intramembrane proteolysis of the RAGE receptor. Biochemical and Biophysical Research Communications 2008; 370: 1-5.

42. Reis CA, David L, Correa P, Carneiro F, De Bolos C, et al. Intestinal metaplasia of human stomach displays distinct patterns of mucin (MUC1, MUC2, MUC5AC, and MUC6) expression. Cancer Res 1990; 59: 1003-7.

43. Baldus SE, Engelmann K, Hanisch FG. MUC1 and the MUCs: a family of human mucins with impact in cancer biology. Crit Rev Clin Lab Sci 2004; 41: 189-231.

44. Udhayakumar G, Jayanthi V, Devaraj N, Devaraj H. Nuclear translocation of $\beta$-catenin correlates with CD44 upregulation in Helicobacter pylori-infected gastric carcinoma. Mol Cell Biochem 2011; 357 (1-2): 283-93. Epub 2011 jun 16.

45. Hirata Y, Maeda S, Mitsuno Y, Akanuma M, Yamaji Y, Ogura K, et al. Helicobacter pylori Activates the Cyclin D1 Gene through Mitogen Activated Protein Kinase Pathway in Gastric Cancer Cells. Infection and immunity 2001; 3965-71.

46. Cho YJ, Kim JH, Yoon J, Cho Sj, Ko YS, Park JW, et al. Constitutive activation of glycogen synthase kinase-3b 
correlates with better prognosis and cyclin-dependent kinase inhibitors in human gastric cancer. BMC Gastroenterology 2010; 10: 91.

47. Carraway KL, Funes M, Workman HC, Sweeney C. Contribution of membrane mucins to tumor progression through modulation of cellular growth signaling pathways. Curr Top Dev Biol 2007; 78: 1-22.

48. Udhayakumar G, et al. Interaction of MUC1 with betacatenin modulates the Wnt target gene cyclinD1 in $H$. pylori-induced gastric cancer. Mol Carcinog 2007; 46 (9): 807-17.

49. Tesarová $\mathrm{P}$, Kalousová $\mathrm{M}$, Jáchymová $\mathrm{M}$, Mestek $\mathrm{O}$, Petruzelka L, Zima T. Receptor for advanced glycation end products (RAGE)-soluble form (sRAGE) and gene polymorphisms in patients with breast cancer. Cancer
Invest 2007; 25 (8): 720-5.

50. Jing R, Cui M, Wang J, Wang H. Receptor for advanced glycation end products (RAGE) soluble form (sRAGE): a new biomarker for lung cancer. Neoplasma 2010; 57 (1): 55-61.

51. Elangovan I, Thirugnanam S, Chen A, Zheng G, Bosland MC, Kajdacsy-Balla A, Gnanasekar M. Targeting receptor for advanced glycation end products (RAGE) expression induces apoptosis and inhibits prostate tumor growth. Biochem Biophys Res Commun 2012; 417 (4): 1133-8. Epub 2011 dec 20.

52. Liang H, Zhong Y, Zhou S, Peng L. Knockdown of RAGE expression inhibits colorectal cancer cell invasion and suppresses angiogenesis in vitro and in vivo. Cancer Letters 2011; 313: 91-8. 